\title{
-Original-
}

\section{Cross-cultural Validation of the Japanese Version of the Lung Cancer Subscale on the Functional Assessment of Cancer Therapy-Lung}

\author{
Erika Saitoh $^{1,2}$, Yuki Yokomizo ${ }^{1}$, Chih-Hung Chang ${ }^{3}$, \\ Sonya Eremenco ${ }^{4}$, Hiyori Kaneko ${ }^{5,6}$ and Kunihiko Kobayashi ${ }^{1,5,6}$ \\ ${ }^{1}$ Department of Respiratory Medicine, Saitama Medical University \\ ${ }^{2}$ Department of Respiratory Medicine, Komagome Hospital, Tokyo \\ ${ }^{3}$ Buehler Center on Aging, Health and Society, Northwestern University Feinberg School of Medicine, Chicago, Illinois, USA \\ ${ }^{4}$ Center on Outcomes, Research and Education, Evanston Nothwestern Healthcare, Evanston, Illinois, USA \\ ${ }^{5}$ Department of Pulmonary Medicine/Infection and Oncology, Graduate School of Medicine, Nippon Medical School \\ ${ }^{6}$ East Japan Chesters Group, Tokyo
}

\begin{abstract}
Background: The Functional Assessment of Cancer Therapy-Lung (FACT-L) questionnaire, which consists of a core questionnaire (the General Measure of FACT [FACTG]) and a 9-item Additional Concerns comprised of a 7-item Lung Cancer Subscale (LCS), was developed in an English-speaking culture. The validation of the Japanese FACT-G was reported previously, and this report describes the cross-cultural validation of the LCS.

Methods: The Japanese version of the LCS was developed through an iterative forwardbackward translation sequence used throughout the FACT Multilingual Translation Project. In evaluating psychometric performance, its construct validity was investigated with Cronbach's alpha coefficient and factor analysis. Clinical validities of a known-groups comparison and longitudinal validity were also investigated.

Results: The FACT-L was administered twice to 180 patients with lung cancer within 2 weeks. The Japanese LCS had borderline values for Cronbach's alpha coefficients $(0.62-0.67)$. Factor analysis indicated that the LCS had the three dimensions of respiratory symptoms, appetite plus body weight, and clear thinking. For clinical validity, a known-groups comparison showed that the LCS could differentiate patients according to truth disclosure, as Japanese doctors sometimes do not fully inform terminally ill patients. However, responsiveness was not proved when performance status was used as an anchor, probably owing to the short interval between the administration of the two measures.

Conclusion: The Japanese version of the LCS asked questions about multiple symptoms of patients with lung cancer, as did the original English LCS. The longitudinal clinical validity of the Japanese version should be investigated in future clinical trials.

(J Nippon Med Sch 2007; 74: 402-408)
\end{abstract}

Key words: cross-cultural comparison, Japanese, lung carcinoma, quality of life, questionnaire, Functional Assessment of Cancer Therapy-Lung, Lung Cancer Subscale

Correspondence to Kunihiko Kobayashi, MD, PhD, Department of Respiratory Medicine, Saitama Medical University, 38 Morohongo, Moroyama Iruma, Saitama 350-0495, Japan

E-mail: kobakuni@saitama-med.ac.jp

Journal Website (http://www.nms.ac.jp/jnms/) 


\section{Introduction}

Most patient-centered questionnaires assessing quality of life (QOL) have been developed in Englishspeaking cultures. Thus, whether these tools are applicable to patients from other cultures has been questioned. Both the Japanese language and culture differ from those of English-speaking countries. In research on cross-cultural validation, whether instruments universally applicable can be validated cross-culturally in Japan is of great interest ${ }^{1}$.

The Functional Assessment of Cancer Therapy (FACT) scale was developed and validated by Cella and colleagues in an English-speaking culture (the United States) over the course of 10 years (19871997) ${ }^{2}$. To determine if the FACT scale could be used in Japan, we undertook a cross-cultural validation. We developed the Japanese version of the General Measure of FACT (FACT-G, version 3) through an iterative forward-backward translation sequence used throughout the FACT multilingual translation project. Previously, we reported the results of psychometric testing for FACT-G ${ }^{3}$, indicating that the scales for Physical, Functional, Emotional Well-Being, and Relationship with Doctors were constructively valid in Japan. However, Japanese patients felt that familial relationships differed from those with friends and neighbors, indicating that the Social/Family Well-Being scale needed to be adjusted owing to cultural differences. Two items concerning coping with illness and acceptance of illness did not load predictably onto their respective scales and were considered to be cross-culturally problematic.

The FACT Measurement System has also included the development of disease/conditionspecific modules, for example, for breast cancer and prostate cancer, as well as symptom-specific subscales, for example, for fatigue. Specific modules aim to investigate special conditions and related QOL issues, for example, body image after mastectomy for patients with breast cancer, incontinence of urine for patients with prostate cancer, and fatigue for cancer patients with anemia. These specific modules are combined with the core questionnaire, FACT-G, and designated as Additional Concerns and designated as FACT-B, FACT-P, or FACT-An, respectively.

We also developed a Japanese version of the FACT-Lung (FACT-L), which includes the FACT-G and Additional Concerns for patients with lung cancer, at the same time that the Japanese FACT-G was created. The 9-item Additional Concerns on FACT-L comprises a 7-item Lung Cancer Subscale (LCS). This report describes the reliability and validity of the LCS and provides fundamental information to Japanese clinical researchers who investigate patients' QOL using FACT-L.

\section{Patients and Methods}

From October 1996 through February 1998, development and validation of the Japanese version of the FACT-L took place in three phases: (1) item generation (translation/back-translation process), (2) pilot-testing (content validity), and (3) estimation of reliability and validity. In all phases the procedures followed were in accordance with the Helsinki Declaration (1964, amended in 1975 and 1983) of the World Medical Association. The results of item generation and pilot-testing were reported previously $^{3}$. The present report focuses on psychometric testing for the Japanese LCS.

\section{Japanese Version of LCS}

Additional Concerns on the FACT-L (version 3) consisted of questions about 9 items: shortness of breath, weight loss, clarity of thinking, cough, hair loss, appetite, chest tightness, difficulty breathing and regret for smoking. A 7-item LCS was made without the items on hair loss and regret for smoking. Severity was assessed using a 0-4 Likert scale. To show the worst condition as 0 , the polarity of scale for some items was reversed, and all item scores for the LCS were totaled to give the LCS score. Therefore, LCS scores ranged from 0 (fully symptomatic) to 28 (asymptomatic).

\section{Study Sample and Protocol}

The subjects were inpatients or outpatients with lung cancer at 8 hospitals within the East Japan 
Chesters Group. After informed consent had been obtained to assess QOL, the Japanese LCS was administered twice to each patient at an interval of 2 weeks. Completed questionnaires were submitted by each patient to our QOL Center (E.P.S. Co., Ltd., Tokyo, Japan) without the participation of the patient's physician. However, the patient's physicans were asked about the diagnosis, stage, Eastern Cooperative Oncology Group (ECOG) performance status (PS), treatment, and degree of disclosure about the diagnosis to the patient.

\section{Psychometric Testing}

Descriptive statistics and reliability. The mean and standard deviation (SD) of the scores for the scales were calculated according to a previously reported method $^{24}$. Including only patients with stable PS and who had not received any therapy within the previous 2 weeks, test-retest reliability was investigated by calculating Pearson's correlation coefficients of items between two measures. The internal consistency of each scale was estimated with Cronbach's alpha coefficient ${ }^{5}$. A value of 0.70 or greater was considered acceptable for group comparison $^{1,3}$.

Factor analysis and validity testing. To investigate the structure of the LCS, factor analysis was performed with Promax rotation using the 7 items. To evaluate the clinical validity of the Japanese LCS, known-group comparison was performed to judge the extent to which the questionnaire scores were able to discriminate between subgroups of patients differing in clinical status. The clinical variables of histologic diagnosis, treatment, cancer disclosure, and outpatient/inpatient status were used to form mutually exclusive patient subgroups. The Wilcoxon test or the Kruskal-Wallis test was used to assess the statistical significance of group differences. Finally, to determine clinical validity, changes in the Japanese LCS score over time were examined in relation to changes in ECOG PS. Improvement or deterioration in a group was defined as a shift of at least 1 level downward or upward, respectively, in ECOG PS. Repeated-measures analysis of variance was used to test intergroup differences in scores over time with the use of the Japanese LCS.

\section{Results}

The purpose of pretesting was to obtain data on the acceptability, appropriateness, and comprehensibility of the instrument. There was no major problem in translating the 9 items of the Additional Concerns. However, one requirement of the retranslations based on pretesting was altering the translations of "not at all," "a little bit," "somewhat," “quite a bit", and "very much" on the Likert scale. Japanese patients often made a mistake in choosing an answer for an item that included a negative phrase. The word "apply" was therefore added to the translation of the responses on the Likert scale as follows: "not apply at all," "apply a little bit," "apply to some degree," "apply quite a bit," and "apply very much."

\section{Descriptive Statistics and Reliability}

Characteristics of the 180 patients with primary lung cancer who participated are shown in Table $\mathbf{1}$. The patients were 136 men and 44 women. Most had progressive disease (stage IIIB, 48 patients; stage IV, 86 patients) and were inpatients (154 patients) but had a good PS (PS 0-2, 173 patients; PS 3-4, 4 patients; data missing from 3 patients). Most of the patients were fully informed of their diagnoses (157 patients were informed; 23 were not informed).

For test-retest reliability for each item on Additional Concerns, Table 2 lists correlation coefficients between the two assessments. Of the 180 patients, only those with unchanged PS and those who received no therapy in the previous 2 weeks were considered. All items on Additional Concerns except for Q6 ("I have a good appetite") and Q9 ("Breathing is easy for me") had correlation coefficients greater than 0.5. Q6 and Q9 might be too sensitive for change.

For score distribution and internal consistency, Table 3 lists means \pm SD and Cronbach's alpha coefficients between the two assessments, respectively. The means \pm SD are from data on 180 patients, with a higher value representing lesssevere symptoms. Cronbach's alpha coefficients for the 9-item Additional Concerns were 0.49 and 0.57 in 
Cross-cultural Validation of FACT-L

Table 1 Patient Characteristics $(n=180)$

\begin{tabular}{lcc}
\hline \multicolumn{1}{c}{ Variable } & Category & $\mathrm{n}$ \\
\hline Sex & Male/Female & $136 / 44$ \\
Age (years) & $\leq 39$ & 3 \\
& $40-49$ & 11 \\
& $50-59$ & 43 \\
& $60-69$ & 67 \\
& $70-79$ & 49 \\
ECOG PS & $\geq 80$ & 5 \\
& 0 & 51 \\
Histologic diagnosis & 1 & 80 \\
Stage & 2 & 42 \\
& 3 & 3 \\
& 4 & 1 \\
Full disclosure of diagnosis & NSCLC/SCLC & $128 / 49$ \\
Patient location & Others & 2 \\
Treatment & I-II & 12 \\
& IIIA & 26 \\
& IIIB & 48 \\
& IV & 86 \\
& Chemo/Radient/Outpatient & $157 / 23$ \\
& Others & $154 / 22$ \\
& None & $38 / 3 / 9$ \\
& & 2 \\
& In & 68 \\
& &
\end{tabular}

Table 2 Test-retest reliability for items

\begin{tabular}{lll}
\hline & & $\mathrm{r}$ \\
\hline Q1 & I have been short of breath & $\mathbf{. 7 3}$ \\
Q2 & I am losing weight & $\mathbf{. 6 8}$ \\
Q3 & My thinking is clear & $\mathbf{. 5 2}$ \\
Q4 & I have been coughing & $\mathbf{. 6 6}$ \\
Q5 & I am bothered by hair loss & $\mathbf{. 6 0}$ \\
Q6 & I have a good appetite & .47 \\
Q7 & I feel tightness in my chest & $\mathbf{. 6 0}$ \\
Q8 & Breathing is easy for me & .33 \\
Q9 & I regret my smoking & $\mathbf{. 7 0}$ \\
\hline
\end{tabular}

Of all the 180 patients with lung cancer, only those cases who had unchanged PS and no therapy over two weeks were considered here $(\mathrm{n}=129)$. The data were analyzed from the 1 st and the 2nd measurements. Correlation coefficients more than 0.5 are indicated using bold numbers, which are considered to indicate test-retest reliability.

the first and second measurements, respectively, indicating unsatisfactory internal consistency within these subscales. On the other hand, borderline internal consistency was obtained for the 7-item LCS. Namely, Q5: “I am bothered by hair loss," and
Q10: "I regret my smoking," were considered to be different from the other 7 items.

\section{Factor Analysis and Validity Testing}

Borderline internal consistency for the 7-item LCS also indicated the possibility that the LCS has multiple factors. Table 4 lists the item-to-factor loadings for the Japanese LCS when the data from all 180 patients were analyzed, and three factors were extracted: Respiratory Symptoms (factor 1), Appetite \& Body Weight (factor 2), and Clear Thinking (factor 3).

Clinical validity, differentiating known groups, is shown in Table 5. Components of the LCS were able to differentiate patients according to histologic diagnosis, full disclosure, and location of the administration of the instrument (inpatient or outpatient). Sensitivity to histologic diagnosis was seen in the Appetite \& Body Weight subscale, indicating significantly worse QOL in patients with non-small cell lung cancer (NSCLC) than in patients with small cell lung cancer (SCLC). In terms of full disclosure, patients who had not been fully informed 
E. Saitoh, et al

Table 3 Reliability analyses

\begin{tabular}{|c|c|c|c|c|}
\hline \multirow[b]{2}{*}{ Subscale } & \multicolumn{4}{|c|}{ Japanese FACT-L $(\mathrm{n}=180)$} \\
\hline & Item No. & $\begin{array}{c}\text { Range of } \\
\text { Scores }\end{array}$ & Mean $\pm \mathrm{SD}$ & $\alpha$ \\
\hline A. C. $*(1) \#$ & $\mathrm{Q} 1-9$ & $0-36$ & $24.1 \pm 6.1$ & .49 \\
\hline A. C. $*(2) \# \#$ & $\mathrm{Q} 1-9$ & $0-36$ & $23.9 \pm 7.0$ & .57 \\
\hline $\operatorname{LCS}(1) \#$ & $\mathrm{Q} 1-4,6-8$ & $0-28$ & $17.81 \pm 5.7$ &.$\overline{62}$ \\
\hline $\operatorname{LCS}(2) \# \#$ & $\mathrm{Q} 1-4,6-8$ & $0-28$ & $17.8 \pm 6.2$ & .68 \\
\hline
\end{tabular}

* A. C.: Additional Concerns on the FACT-L

\#: Data were from the 1st measurement.

\#\#: Data were from the 2nd measurement.

$\alpha$ : Cronbach's alpha

A value of 0.70 or greater of Cronbach's alpha indicates good internal consistency.

Cronbach's alpha coefficients for the 9-item A. C. indicated unsatisfactory internal consistency within these subscales. On the other hand, the borderline internal consistency was obtained for the 7-item LCS.

Table 4 Factor Analysis with Promax Rotation of the 7-item LCS $(n=180)$

\begin{tabular}{cccc}
\hline Item & Factor 1 & Factor 2 & Factor 3 \\
\hline Q1 & $\mathbf{. 8 0}$ & .01 & -.01 \\
Q4 & $\mathbf{. 5 8}$ & -.00 &.$- \mathbf{4 2}$ \\
Q7 & $\mathbf{. 7 6}$ & -.03 & .12 \\
Q9 & $\mathbf{. 6 0}$ & -.05 & .51 \\
Q2 & .24 &. $\mathbf{7 6}$ & -.20 \\
Q6 & -.13 & .89 & .23 \\
Q3 & .03 & .00 &. $\mathbf{8 5}$ \\
\hline
\end{tabular}

The values of factor loading more than 0.40 are bold. Factor Analysis on 7-item LCS indicated the three factors of Respiratory Symptoms (factor 1), Appetite \& Body Weight (factor 2), and Clear Thinking (factor 3). The 7-item LCS asked about multiple symptoms of patients with lung cancer.

of their diagnoses lost appetite and body weight as Japanese doctors sometimes do not fully inform terminally ill patients. The comparison across patient location showed that outpatients had better QOL in Appetite \& Body Weight than did inpatients.

At the subsequent assessment 2 weeks later, ECOG PS had improved by more than 1 point in 9 patients, had deteriorated in PS by more than 1 point in 7 patients, and had remained unchanged in 114 patients. Repeated-measures analysis of variance was employed to test for intergroup differences over time. As the numbers of patients who showed improvement or deterioration were small, statistically significant intergroup differences over time were not observed for the LCS (Table 6).

\section{Discussion}

Most patients with lung cancer present with advanced disease, which is associated with a poor prognosis and symptoms such as pain, coughing, and shortness of breath. Even if patients are at an earlier stage, the progressive nature of lung cancer and its resistance to treatment often result in recurrence with the associated symptoms of advanced disease. These symptoms negatively affect QOL and PS. There is increasing interest in using assessments of improvements in symptoms and QOL as outcomes in clinical trials for patients with advanced lung cancer.

In the Japanese version of Additional Concerns on the FACT-L, Cronbach's alpha coefficients were unacceptable $(<0.7)$, indicating that the Additional Concerns asked multiple aspects to patients with lung cancer. Even in the LCS, Cronbach's alpha coefficients were borderline, and factor analysis showed that the LCS had the three factors of respiratory symptoms, appetite/weight loss, and clarity of thinking. Specifically, the Japanese version of the LCS asked about many symptoms in patients 
Cross-cultural Validation of FACT-L

Table 5 Known-groups Comparison

\begin{tabular}{lcccc}
\hline & \multicolumn{4}{c}{ LCS Subscale (mean) } \\
\cline { 2 - 5 } & $\mathrm{n}$ & $\begin{array}{c}\text { Respiratory } \\
\text { Symptoms }\end{array}$ & $\begin{array}{c}\text { Appetite \& } \\
\text { Body Weight }\end{array}$ & LCS \\
\hline Histologic diagnosis & & & & \\
SCLC & 46 & 10.6 & 4.9 & 18.7 \\
NSCLC & 117 & 10.5 & 4.4 & 17.5 \\
p & & NS & $\underline{0.044}$ & NS \\
Treatment & & & & \\
None & 61 & 10.4 & 4.6 & 18.1 \\
ChemoTx & 38 & 10.4 & 4.3 & 17.4 \\
Radiation & 3 & 12.0 & 3.7 & 16.9 \\
ChemoRT & 7 & 11.1 & 5.0 & 19.6 \\
p & & NS & NS & NS \\
Disclosure & & & & \\
Lung cancer & 144 & 10.7 & 4.7 & 18.1 \\
No disclosure & 22 & 9.4 & 3.2 & 15.4 \\
p & & NS & $\underline{0.012}$ & $\underline{0.023}$ \\
Location & & & & \\
inpatient & 145 & 10.8 & 4.3 & 17.8 \\
outpatient & 18 & 8.7 & 6.0 & 18.5 \\
p & & NS & $\underline{0.006}$ & NS \\
\hline
\end{tabular}

To evaluate the clinical validity of the 7-item LCS, the known-group comparison was performed to judge the extent to which the questionnaire scores were able to discriminate between subgroups of patients differing in clinical status. The clinical variables of histologic diagnosis, treatments, cancer disclosure, and outpatient/inpatient status were used. P values less than 0.05 by Wilcoxon test or Kruskal-Wallis test are underlined.

Table 6 Preliminary Results of Sensitivity to Change in PS

\begin{tabular}{lrccc}
\hline \multirow{2}{*}{ PS } & $\mathrm{n}$ & \multicolumn{3}{c}{ FACT-L Subscale (mean \pm SD) } \\
\cline { 3 - 5 } & & Additional Concerns & Appetite \& Body Weight & LCS \\
\hline Decreased PS & 7 & $0.29 \pm 2.29$ & $0.86 \pm 2.27$ & $0.57 \pm 2.70$ \\
No change & 114 & $0.35 \pm 2.44$ & $0.04 \pm 1.88$ & $0.35 \pm 3.31$ \\
Improved PS & 9 & $1.44 \pm 2.51$ & $-0.67 \pm 0.87$ & $0.33 \pm 2.24$ \\
F value & & 0.85 & 1.34 & 0.02 \\
P value & & 0.4298 & 0.2660 & 0.9843 \\
\hline
\end{tabular}

To determine longitudinal clinical validity, changes in the FACT-L Subscale scores over time were examined in relation to changes in ECOG PS. As the numbers of patients showing improvement or deterioration were small, statistically significant intergroup differences over time were not observed.

with lung cancer. This was the same as in the original English FACT-L. Cronbach's alpha coefficients for English Additional Concerns and LCS were 0.53 and 0.68 , respectively ${ }^{4}$. Both the original and Japanese LCS are reliable patient questionnaires that evaluate and quantify QOL across several dimensions, including lung cancer-related symptoms.

Concerning the clinical validity for the Japanese FACT-L, a known-groups comparison showed that components of the LCS were able to differentiate patients according to histologic diagnosis, full disclosure of diagnosis, and location of 
administration. In longitudinally clinical validity, however, responsiveness was not proven when PS was used as an anchor. This result might be due to limited number of patients and the short interval between administration of the two measures. On the other hand, the English FACT-L has been used in numerous clinical studies, especially those investigating molecularly targeted tyrosine kinase inhibitors $^{6-9}$. In the Iressa Dose Evaluation in Advanced Lung Cancer (IDEAL)-1 and IDEAL-2 phase II monotherapy trials ${ }^{10,11}$, FACT-L showed that treatment of patients who had advanced NSCLC with the tyrosine kinase inhibitor gefitinib resulted in rapid improvements in NSCLC-related symptoms and QOL. Improvements in NSCLC-related symptoms and QOL, as measured with FACT-L, correlated with tumor response, and improvements in symptoms also correlated with progression-free and overall survival. Whether the LCS indicates clinically meaningful changes has been investigated in clinical studies ${ }^{6.9}$. A 2-point change in the total LCS score, which ranges from 0 (severe debilitation) to 28 (asymptomatic), has been shown to correlate with both survival and performance status ${ }^{6-9,12}$.

In conclusion, the Japanese version of the LCS can be used to evaluate multiple symptoms in Japanese patients with lung cancer as can the original English LCS. In Japan, the LCS alone or a 21-item Trial Outcome Index, which combines the scores of the Physical Well-Being and Functional Well-Being scales and the LCS, is recommended for use in clinical studies because the Japanese version of the Social/Family Well-Being scale is somewhat problematic. Although the original LCS scale is a sensitive disease-specific module used to evaluate new targeted therapies, the next step in development of the Japanese LCS will be a test of its longitudinal clinical validity in a clinical trial investigating molecularly targeted drugs, such as tyrosine kinase inhibitors.

\section{References}

1. Kobayashi K, Takeda F, Teramukai S, et al.: A cross- validation of the European Organization for Research and Treatment of Cancer QLQ-C30 (EORTC QLQ-C30) for Japanese with lung cancer. Eur J Cancer 1998; 34: 810-815.

2. Cella DF, Tulsky DS, Gray G, et al.: The Functional Assessment of Cancer Therapy scale: development and validation of the general measure. $\mathrm{J}$ Clin Oncol 1993; 11: 570-579.

3. Fumimoto $\mathrm{H}$, Kobayashi $\mathrm{K}$, Chang $\mathrm{CH}$, et al: Crosscultural validation of an international questionnaire, the General Measure of the Functional Assessment of Cancer Therapy scale (FACT-G), for Japanese. Qual Life Res 2001; 10: 701-709.

4. Cella DF, Bonomi AE, Lloyd SR, Tulsky DS, Kaplan E, Bonomi P: Reliability and validity of the Functional Assessment of Cancer Therapy-Lung (FACT-L) quality of life instrument. Lung Cancer 1995; 12: 199-220.

5. Cronbach LJ: Coefficient alpha and the internal structure of tests. Psychometrika 1951; 16: 297.

6. Cella D, Eton DT, Fairclough DL, et al.: What is a clinically meaningful change on the Functional Assessment of Cancer Therapy-Lung (FACT-L) Questionnaire? Results from Eastern Cooperative Oncology Group (ECOG) Study 5592. J Clin Epidemiol 2002; 55: 285-295.

7. LoRusso PM, Herbst RS, Rischin D, et al.: Improvements in quality of life and disease-related symptoms in phase I trials of the selective oral epidermal growth factor receptor tyrosine kinase inhibitor ZD1839 in non-small cell lung cancer and other solid tumors. Clin Cancer Res 2003; 9: 20402048.

8. Mark G, Ronald B, Roy S, et al.: Efficacy of Gefitinib, an Inhibitor of the Epidermal Growth Factor Receptor Tyrosine Kinase, in Symptomatic Patients With Non-Small Cell Lung Cancer A Randomized Trial. JAMA 2003; 290: 2149-2158.

9. Cella D, Herbst RS, Lynch TJ, et al.: Clinically meaningful improvement in symptoms and quality of life for patients with non-small-cell lung cancer receiving gefitinib in a randomized controlled trial. J Clin Oncol 2005; 23: 2946-2954.

10. Fukuoka M, Yano S, Giaccone G, et al: Multiinstitutional randomized phase II trial of gefitinib for previously treated patients with advanced non-smallcell lung cancer (The IDEAL 1 Trial). J Clin Oncol 2003; 21: 2237-2246.

11. Kris MG, Natale RB, Herbst RS, et al: Efficacy of gefitinib, an inhibitor of the epidermal growth factor receptor tyrosine kinase, in symptomatic patients with non-small cell lung cancer: a randomised trial. JAMA 2003; 290: 2149-2158.

12. Cella D: Impact of ZD1839 on non-small cell lung cancer-related symptoms as measured by the functional assessment of cancer therapy-lung scale. Semin Oncol 2003; 30 (1 Suppl 1): 39-48.

(Received,

April 17, 2007)

(Accepted, September 18, 2007) 